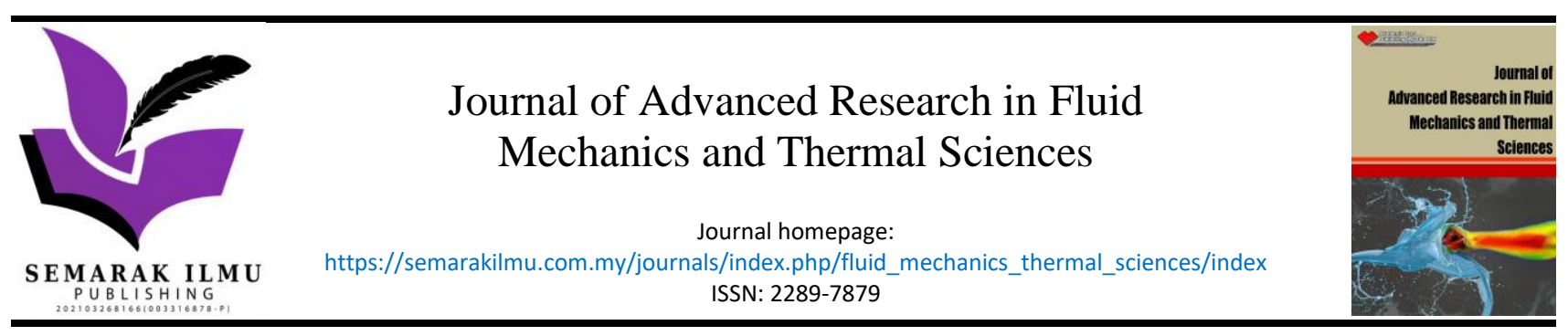

\title{
Slip Effect on Stagnation Point Flow and Heat Transfer Over a Shrinking/Stretching Sheet in A Porous Medium with Suction/Injection
}

\author{
Nirwana Japili ${ }^{1}$, Haliza Rosali ${ }^{*}{ }^{*}$, Norfifah Bachok ${ }^{1,2}$ \\ Department of Mathematics, Faculty of Science, Universiti Putra Malaysia, 43400 UPM Serdang, Selangor, Malaysia \\ Institute for Mathematical Research, Universiti Putra Malaysia, 43400 UPM Serdang, Selangor, Malaysia
}

\section{ARTICLE INFO}

\section{Article history:}

Received 24 August 2021

Received in revised form 3 December 2021

Accepted 10 December 2021

Available online 3 January 2022

\section{Keywords:}

Slip; suction/injection; stagnation point flow; stretching/shrinking sheet; porous medium

\section{ABSTRACT}

The effects of thermal and velocity slip on Newtonian fluid steady boundary layer stagnation-point flow and heat transfer through a porous shrinking sheet with suction effect are investigated in the present research. By using a similarity transformation, the partial differential equation (PDE) is converted to an ordinary differential equation (ODE) and solved using the shooting technique to get numerical results. The flow characteristics for various values of the physical parameters are discussed, taking into consideration the effects of suction/injection as well as velocity and thermal slip. The local Nusselt number and skin friction coefficient, as well as the temperature and the velocity profiles, are presented as results. It is noticed that when the suction and velocity slip increase, the skin friction coefficient and local Nusselt number rise, as does the range of solutions that exist.

\section{Introduction}

Stagnation flow is a term that refers to the movement of fluid near its stagnation point. Hiemenz initiated research on two-dimensional steady stagnation point flow in 1911 when he found an accurate solution by converting the Navier-Stokes equations to the nonlinear ordinary differential equations through similarity transformation, as detailed in Chamkha and Khaled [1]. Numerous academics have since researched stagnation point flow, also known as Hiemenz flow, which Howarth improved in 1935. Various scholars have sought to apply the concept of stagnation point flow to a number of physical phenomena, including shrinking or stretching sheets.

Crane [2] was the first researcher to examine the subject of stagnation point flow on a linearly stretching sheet and to discover the problem's closed analytical solution. Then, by adding suction or injection on the surface, Gupta and Gupta [3] further investigated Crane's problem. While Wang [4] discovered the axisymmetric case's similarity solutions. Bhattacharyya and Gupta [5] then examined the stability of the Crane problem. Chiam [6] then combined the Hiemenz and Crane problems of stagnation point flow in two-dimensional by examining heat transport through a viscous fluid

\footnotetext{
* Corresponding author.

E-mail address: liza_r@upm.edu.my

https://doi.org/10.37934/arfmts.90.2.7389
} 
approaching a stretched plate. Mahapatra and Gupta [7] expanded on Chiam's work by including stagnation point flows and a stretched sheet with varying free flow velocities. They discovered two distinct boundary layers close to the sheet, each with a different ratio of stretching to straining rates. Additionally, Ishak et al., [8] examined the behaviour of the flow over a stretching sheet under a variety of physical conditions while Layek et al., [9] investigated heat generation and suction effect. Nadeem et al., [10] investigated the Homotopy Analysis Method solution for the problem of boundary layer and Mustafa et al., [11] investigated different fluid that is nanofluid in their study.

A lot of researchers investigated the stretching case, whereas just a few dealt with the shrinking case. Ishak et al., [12] considered a micropolar fluid, whereas Bachok et al., [13] addressed the problem using a nanofluid. In addition, Bhattacharya et al., [14] investigated the flow and the heat transfer over a shrinking sheet while taking slip effect into account. Later, Bhattacharya and Layek [15] considered the suction/blowing effect to examine fluid flow, taking thermal radiation into account and expanding their research to include chemical reactions (see Bhattacharya [16]). Moreover, Bhattacharya $[17,18]$ considered the flow in an unsteady case and reveals that when unsteadiness exists, heat transfer rises with the Prandtl number. They then extend their research by including other physical effects such as suction and heat radiation (see Bhattacharya et al., [19]). It is discovered that thermal radiation has an effect on the thermal boundary layer thickness, causing it to become thinner. Following that Bhattacharya and Vajravelu [20] examined the fluid flow over an exponential shrinking case and discovered that increasing the velocity ratio parameter increases the heat transfer rate. Furthermore, Bhattacharya et al., [21,22] extend their prior work by investigating another type of fluid, Casson fluid, and discovered that the similarity solution is identical to that of a Newtonian fluid. Bhattacharya $[23,24]$ conducted more research on the flow of fluid in a shrinking sheet by including non-uniform heat flux and Soret and Dufour effects. While, Aman et al., [25] studied different flows which are magnetohydrodynamics flow with slip effect in their research. One of the significant findings of this study is that when the slip effect is considered, heat transfer rises while skin friction reduces while dual solutions are obtained.

Moreover, the research of shrinking sheets through porous media has garnered attention from many researchers. Rosali et al., [26] studied the flow of the fluid over a porous medium and observed that reducing the porous medium's porosity widen the domain of solutions to exist. Moreover, when Pal and Mandal [27] investigated the flow in a porous medium, they took heat generation into account. They discovered that the skin friction coefficient drops while the local Nusselt number increases when porosity rises. Following that, Bakar et al., [28] explored the Darcy-Forchheimer porous media model in their work. While, Aurangzaib et al., [29] studied the MHD flow of a permeable shrinking sheet with the effect of slip. Then, Nasir et al., [30] considered the flow in the presence of suction via a porous quadratically shrinking or stretching sheet. The increase in suction strength increases the skin friction as well as the heat transfer rate. Numerous scholars, notably Kumar and Sood [31], have conducted more research on porous media. They performed the numerical analysis on nonlinear shrinking cases involving a porous medium. Later, Shomali et al., [32] analyzed the unsteady flow of a nanofluid in porous media and discovered that as the velocity slip changes, the skin friction rises. Then, Jamaludin and Nazar [33] further investigated an exponential case in a porous medium and discovered that an exponential case has a larger possible solution compared to the linear case. Moreover, Japili et al., [34] investigated the flow of the fluid in an exponential case by taking the suction effect into account through a porous medium. It is concluded that the exponential case has a greater solution domain than the linear case. Whereas, Rosali et al., [35] extend the prior work in an unsteady case with slip effect imposed at the surface. Norzawary et al., [36] recently explored the flow of fluids with the impact of suction or injection through a stretching/shrinking sheet of a carbon nanotube. 
According to the literature, most of the research concentrated on fluid flow and heat transfer without considering the influence of slip and suction, specifically in porous media. Thus, further study is required, since certain numerical analyses indicate that these factors may enhance heat exchange in the fluid while simultaneously increasing its velocity. Moreover, some study shows that the suction effect may contribute to the reduction of drag and energy losses in channels. Therefore, numerical studies should be conducted to verify all of these possibilities to provide more exact results. Hence, this research is a continuation of Rosali et al., [26], in which we investigate the stagnation point flow through a porous medium while accounting for the slip and suction effect. We obtain and discuss the findings with different values of physical parameters for the skin friction coefficient and the local Nusselt number and in order to ensure the results' validity, the profiles for temperature and velocity are presented. To our best of knowledge, the results of this paper are new and they have not been published before.

\section{Methodology}

We investigate a continuous stagnation point flow towards a shrinking/stretching sheet in the existence of slip and suction effect through a porous medium. As seen in Figure 1 , the $x$-axis is parallel to and the $y$-axis is perpendicular to the shrinking/stretching sheet. $U_{\infty}(x)=a x(a>0)$ denotes the external flow's velocity which represents the intensity of the stagnation flow. While $U_{w}(x)=b x$ defines the sheet's velocity, where $b<0$ and $b>0$ denoted the shrinking and stretching sheet, respectively.

The temperature of the surface and the ambient fluid are represented as $T_{w}$ and $T_{\infty}$ which are both considered to be constant. Hence, the problem's boundary layer systems may be defined as Rosali et al., [26]

$\frac{\partial u}{\partial x}+\frac{\partial v}{\partial y}=0$

$u \frac{\partial u}{\partial x}+v \frac{\partial u}{\partial y}=U_{\infty} \frac{d U_{\infty}}{d x}+v \frac{\partial^{2} u}{\partial y^{2}}+\frac{v}{K_{1}}\left(U_{\infty}-u\right)$

$u \frac{\partial T}{\partial x}+v \frac{\partial T}{\partial y}=\alpha \frac{\partial^{2} T}{\partial y^{2}}$

in which $u$ and $v$ denote the velocity of $x$ - and $y$-axes. Whereas $K_{1}$ referred to the permeability parameter, $v$ referred to the kinematic viscosity, $T$ referred to the fluid's temperature, $\alpha$ referred to the thermal diffusivity, $L$ and $S$ referred to the slip length and the proportionality constant, respectively. The boundary conditions were determined in accordance with Aman et al., [25], who defined them as below

$$
\begin{aligned}
& v=V_{w}, u=U_{w}(x)+L \frac{\partial u}{\partial y}, T=T_{w}+S \frac{\partial T}{\partial y} \text { at } y=0 \\
& u \rightarrow U_{\infty}(x), T \rightarrow T_{\infty} \text { as } y \rightarrow \infty
\end{aligned}
$$

The following similarity transformation with respect to the boundary condition (4) is used to solve the system of PDEs (1)-(3) 


$$
\begin{aligned}
& \eta=\left(\frac{U_{\infty} x}{\alpha}\right)^{\frac{1}{2}} \frac{y}{x} \\
& \psi=\left(\alpha x U_{\infty}\right)^{\frac{1}{2}} f(\eta) \\
& \theta(\eta)=\frac{T-T_{\infty}}{T_{w}-T_{\infty}}
\end{aligned}
$$

given that $\psi$ is the stream function in which $u=\partial \psi / \partial y$ and $v=-\partial \psi / \partial x$. By substituting Eq. (5) into (1)-(4), we get the system of ODEs as follows

$$
\begin{aligned}
& \operatorname{Pr} f^{\prime \prime \prime}+f f^{\prime \prime}+1-f^{2}+K\left(1-f^{\prime}\right)=0 \\
& \theta^{\prime \prime}+f \theta^{\prime}=0
\end{aligned}
$$

subject to the boundary conditions

$$
\begin{aligned}
& f(0)=s, f^{\prime}(0)=c+A f^{\prime \prime}(0), \theta(0)=1+B \theta^{\prime}(0) \\
& f^{\prime}(\eta) \rightarrow 1, \theta(\eta) \rightarrow 0 \text { as } \eta \rightarrow \infty
\end{aligned}
$$

where prime here signifies differentiation with respect to $\eta . K=v /\left(a K_{1}\right)$ denotes the permeability parameter while $\mathrm{Pr}=v / \alpha$ represents the Prandtl number. Whereas, the coefficient of mass transfer is defined as $s=-V_{w} / \sqrt{a \alpha}$, with suction and injection specified as $s>0$ and $s<0$, respectively. The parameter $c$, which is defined as $c=b / a$, refers to stretching when $c>0$ and shrinking when $c<0 . A=L \sqrt{a \alpha}$ gives the parameter of velocity slip, while $B=S \sqrt{a \alpha}$ gives the parameter of thermal slip. The skin friction coefficients and the local Nusselt number may be represented as

$$
C_{f}=\frac{\tau_{w}}{\rho U_{\infty}^{2} / 2}, N u_{x}=\frac{x q_{w}}{k\left(T_{w}-T_{\infty}\right)}
$$

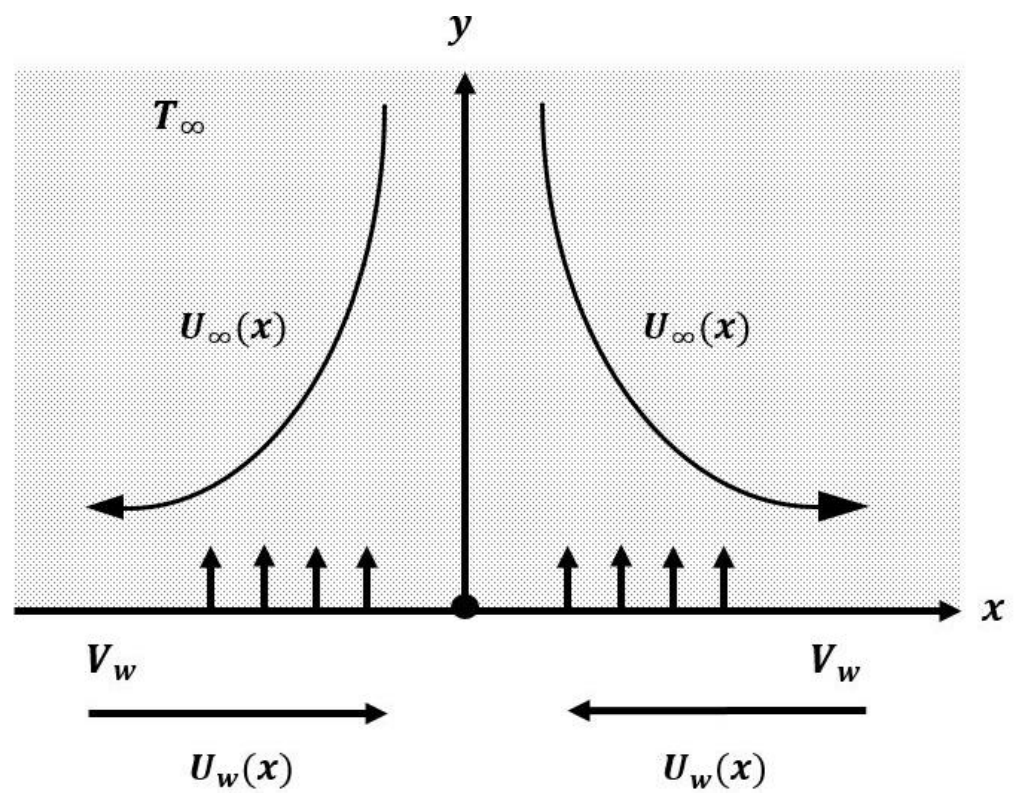

Fig. 1. Physical model of two-dimensional stagnation point flow towards a shrinking sheet with slip effect 
where $\tau_{w}$ and $q_{w}$ represent the shear stress and the heat flux, respectively

$\tau_{w}=\mu\left(\frac{\partial u}{\partial y}\right)_{y=0}, q_{w}=-k\left(\frac{\partial T}{\partial y}\right)_{y=0}$

with $k$ and $\mu$ denoting the coefficients of the thermal conductivity and viscosity, respectively. We find the following expressions by applying the similarity variables defined in Eq. (5)

$\frac{1}{2} C_{f} \operatorname{Re}_{x^{\frac{1}{2}}}=\operatorname{Pr}^{\frac{1}{2}} f^{\prime \prime}(0)$

$N u_{x}=-P e_{x}^{\frac{1}{2}} \theta^{\prime}(0)$

where $R e_{x}=U_{\infty} x / v$ represents the local Reynolds number while $P e_{x}=U_{\infty} x / \alpha$ denotes the local Péclet number.

\section{Results and Discussion}

The ODEs in Eq. (6) and (7) that meet the conditions in Eq. (4) were numerically solved in Maple software using a shooting technique. This study examined the impact of suction/injection parameter $s$, velocity slip parameter $A$, thermal slip parameter $B$ and permeability parameter $K$ on the skin friction coefficient $f^{\prime \prime}(0)$ and local Nusselt number $-\theta^{\prime}(0)$. The study findings are compared to those of Wang [4] and Rosali and Ishak [29] in the absence of permeability parameters as well as slip and suction effect when $\mathrm{Pr}=1$. The present findings are consistent to five decimal places with those reported previously by Wang [4] and Rosali and Ishak [37]. As shown in Table 1, the results indicated a high level of agreement. Variation of the $f^{\prime \prime}(0)$ and the $-\theta^{\prime}(0)$ correspond to the stretching/shrinking parameter $c$ for several suction parameter values are depicted in Figure 2 and 3 , respectively. As illustrated in Figure 2 and 3, there is a dual solution to Eq. (6) and (7) with the boundary conditions specified in Eq. (4) since there is an existence of a critical value $c_{c}$. As illustrated in Figure 2 and 3, dual solutions occur when $c_{c}<c \leq-1.5$, unique solution occurs when $c>-1.5$ and no solution occurs for $c<c_{c}$. Table 2 summarises the critical values $c_{c}$ for different values of $s$. For a given $c$, the first solution in Figure 2 has a greater $f^{\prime \prime}(0)$ value than the second solution. It is also demonstrated that as $s$ increases, so does the magnitude of $f^{\prime \prime}(0)$. The suction causes a rise in the shear stress which raises the velocity gradient near the surface. Additionally, as the suction parameter $s$ increases, the critical value $c_{c}$ also increases, resulting in a broader range of $c$ at which the solutions exist. Besides, the suction effect minimizes drag between fluid and surfaces, delaying the separation of boundary layers.

Moreover, when the value of $s$ rises, so does the magnitude of $-\theta^{\prime}(0)$, indicating an increase in the temperature gradient and hence an increase in the rate of heat transfer. Whereas, Figure 4 and 5 demonstrate the influence of injection parameter $(s<0)$ towards $f^{\prime \prime}(0)$ and $-\theta^{\prime}(0)$ respectively. These figures show that the increase in the injection effect will reduce both $f^{\prime \prime}(0)$ and $-\theta^{\prime}(0)$. Besides, in the shrinking case, there are dual solutions for a given injection parameter value. However, as the injection parameter reached a certain value, a unique solution occurs for any value of $c$. It is also worth noting that the solutions domain is greater in the suction case as compared to the injection case. 


\section{Table 1}

Comparison of the values of $f^{\prime \prime}(0)$ for different values of $c$ when $\operatorname{Pr}=1$ and $s=K=A=B=0$

\begin{tabular}{llll}
\hline$c$ & Wang [1] & Rosali and Ishak [37] & Present Results \\
\hline-1.2465 & 0.55430 & 0.584295 & 0.554283 \\
-1.15 & 1.08223 & 1.082231 & 1.082244 \\
-1 & 1.32882 & 1.328817 & 1.328819 \\
-0.75 & 1.48930 & 1.489298 & 1.489299 \\
-0.5 & 1.49567 & 1.495670 & 1.495670 \\
-0.25 & 1.40224 & 1.402241 & 1.402241 \\
0 & 1.232588 & 1.232588 & 1.232588 \\
0.1 & 1.14656 & 1.146561 & 1.146561 \\
0.2 & 1.05113 & 1.051130 & 1.051130 \\
0.5 & 0.71330 & 0.713295 & 0.713294 \\
2 & -1.88731 & -1.887307 & -1.887307 \\
5 & -10.26475 & -10.264749 & -10.264749 \\
\hline
\end{tabular}

\section{Table 2}

\begin{tabular}{ll} 
Values of $c_{C}$ for different values of $s$ when $\operatorname{Pr}=1$ and $K=A=B=0.5$ \\
\hline$S$ & $c_{C}$ \\
\hline-0.8 & -1.7121 \\
-0.5 & -1.8808 \\
-0.3 & -2.0246 \\
0.3 & -2.6268 \\
0.5 & -2.8887 \\
0.8 & -2.3454 \\
\hline
\end{tabular}

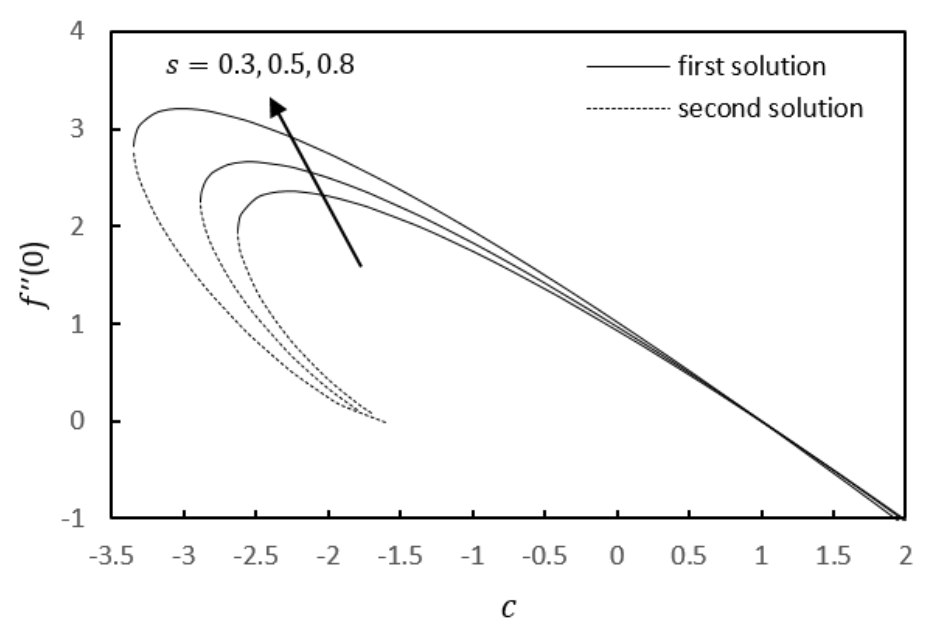

Fig. 2. Skin friction coefficient $f^{\prime \prime}(0)$ with $c$ when $\operatorname{Pr}=1$ and $K=A=B=0.5$ 


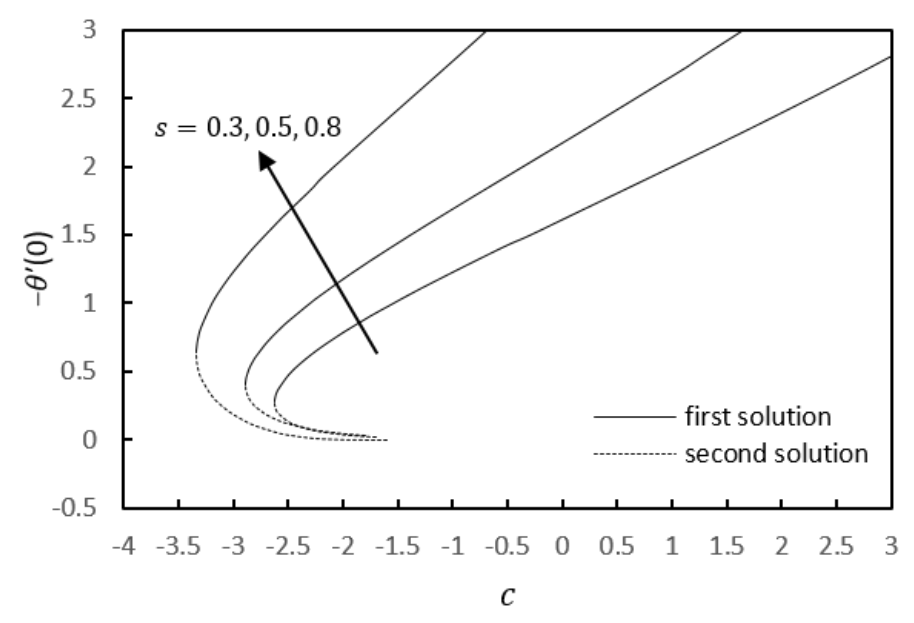

Fig. 3. Local Nusselt number $-\theta^{\prime}(0)$ with $c$ when $\operatorname{Pr}=$ 1 and $K=A=B=0.5$

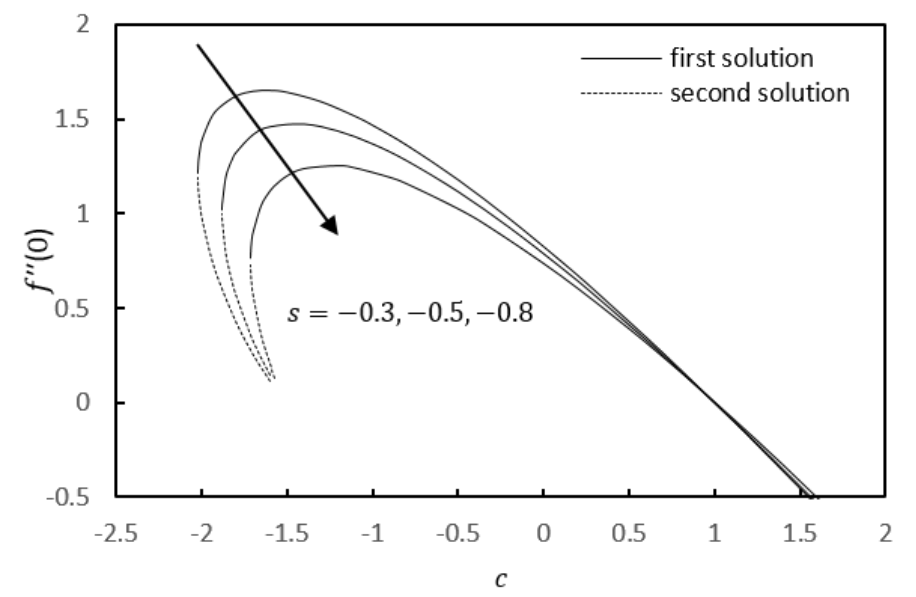

Fig. 4. Skin friction coefficient $f^{\prime \prime}(0)$ with $c$ when $\operatorname{Pr}=1$ and $K=A=B=0.5$

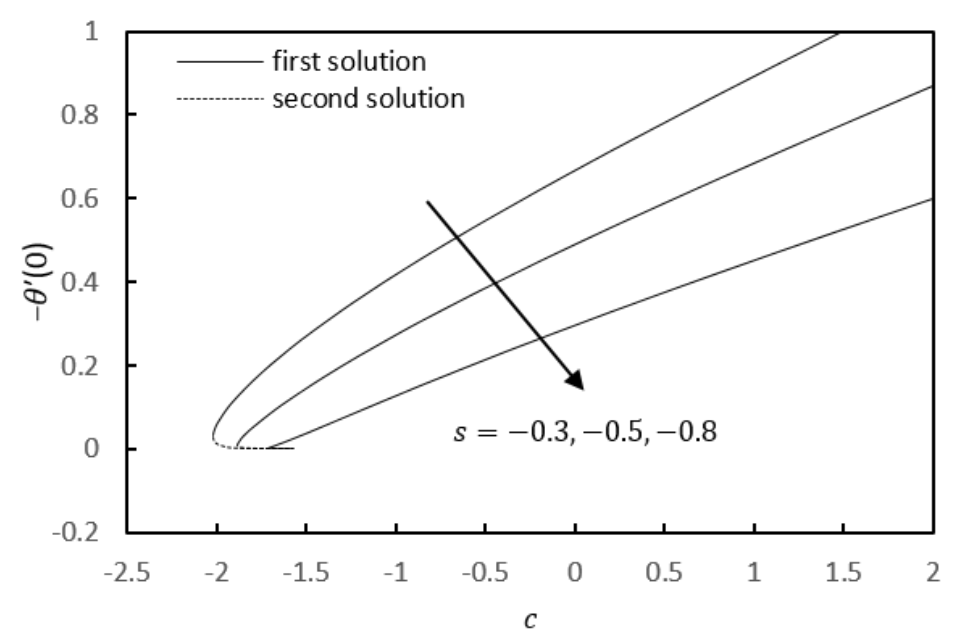

Fig. 5. Local Nusselt number $-\theta^{\prime}(0)$ with $c$ when $\operatorname{Pr}=$ 1 and $K=A=B=0.5$ 
The effect of parameter $A$ on $f^{\prime \prime}(0)$ and $-\theta^{\prime}(0)$ are depicted in Figure 6 and 7, correspondingly. As seen in these figures, there exist both unique and dual solutions. Besides, when the value of $A$ rises, it reduces the value of $f^{\prime \prime}(0)$ but raises $-\theta^{\prime}(0)$, as seen in Figure 6 and 7 , respectively. It has been demonstrated that when velocity slip rises, the solutions domain widens. If the velocity slip effect is taken into account, it indicates that there is a relative movement between the fluid and the surfaces. So, when the value of $A$ increases, it denotes that the fluid's velocity is greater than the surface's velocity. As a result, the existence of the velocity slip effect will enhance the fluid flow, delaying the separation of the boundary layers. This explains why the velocity slip parameter with a greater value has a larger value of $c_{c}$. However, as illustrated in Figure 8 and 9 , the impact of the thermal slip $B$ on $f^{\prime \prime}(0)$ and $-\theta^{\prime}(0)$ was found to be different. The change in $B$ has no influence on the variations of $f^{\prime \prime}(0)$ caused by the decoupling of Eq. (6) and (7), as seen in Figure 8 . When parameter $B$ is decreased, it is observed that the temperature lowers as well, but it has no effect on the variation of the critical value. Therefore, it is clear that the thermal slip has no impact on boundary layer separation.

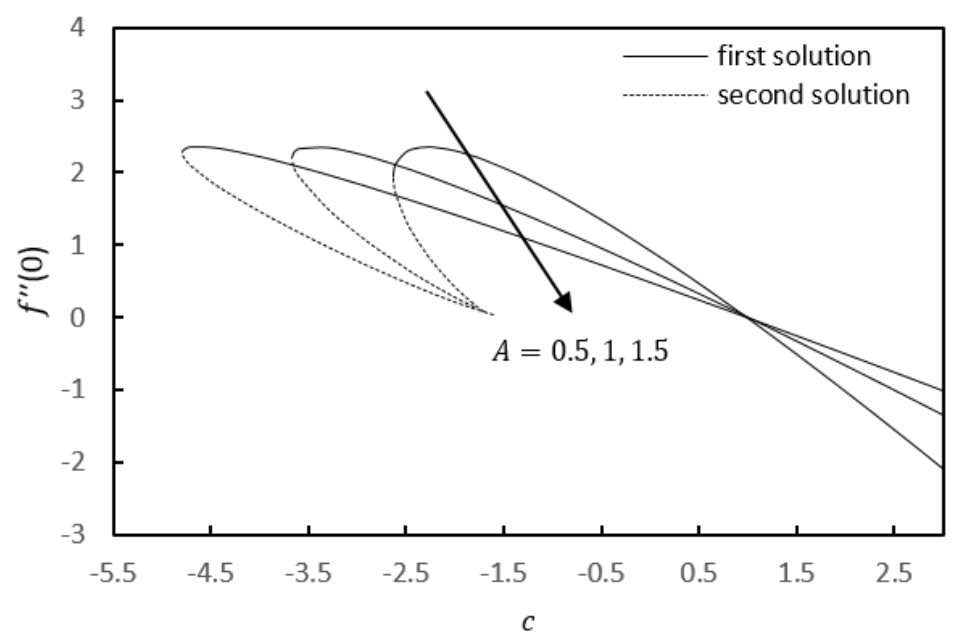

Fig. 6. Skin friction coefficient $f^{\prime \prime}(0)$ with $c$ when $\operatorname{Pr}=1$, $s=0.3$ and $K=B=0.5$

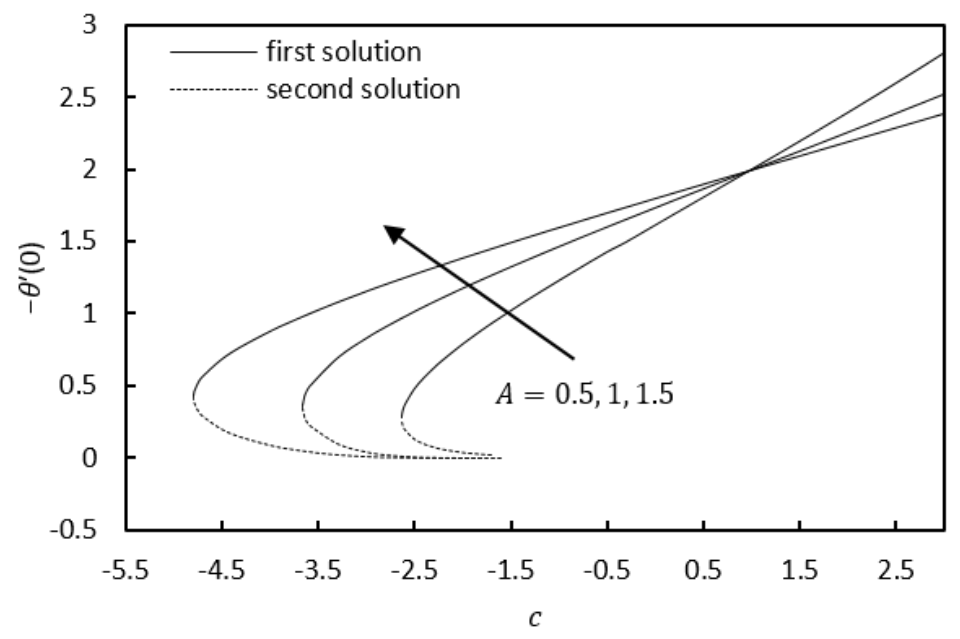

Fig. 7. Local Nusselt number $-\theta^{\prime}(0)$ with $c$ when $\operatorname{Pr}=1$, $s=0.3$ and $K=B=0.5$ 


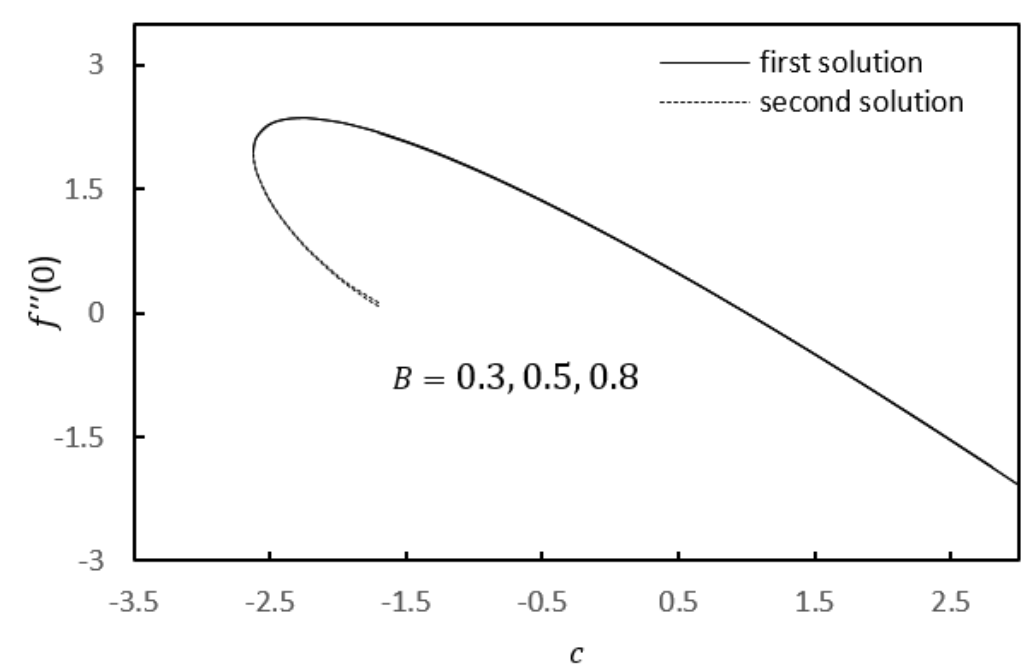

Fig. 8. Skin friction coefficient $f^{\prime \prime}(0)$ with $c$ when $\operatorname{Pr}=1, s=$ 0.3 and $K=A=0.5$

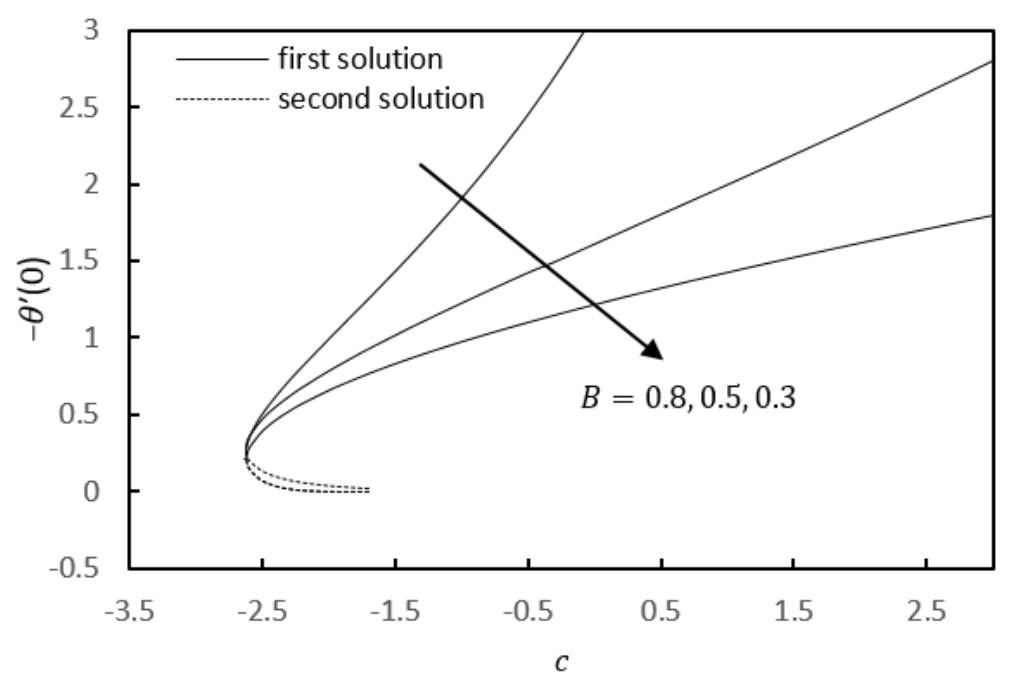

Fig. 9. Local Nusselt number $-\theta^{\prime}(0)$ with $c$ when $\operatorname{Pr}=1, s=$ 0.3 and $K=A=0.5$

Furthermore, the influence of permeability parameter $K$ towards $f^{\prime \prime}(0)$ and $-\theta^{\prime}(0)$ are illustrated in Figure 10 and 11 , respectively. Both the $f^{\prime \prime}(0)$ and $-\theta^{\prime}(0)$ rise as the permeability parameter increases as seen in Figure 10 and 11. Moreover, these figures show that decreasing the porosity of the porous medium, which means a higher permeability parameter value, broadens the range of solutions to Eq. (6) and (7). As can be seen, the impact of combining porosity, velocity slip and suction significantly expands the solutions domain, resulting in a delay in boundary layer separation. Figure 12 and 13 show the suction effect on the velocity $f^{\prime}(\eta)$ and temperature profiles $\theta(\eta)$ of the shrinking case, respectively. Suction $s$ has been shown to influence the velocity profile $f^{\prime}(\eta)$ in the shrinking case, as seen in Figure 12. When $s$ is increased, the velocity profile for the first solution rises while it falls for the second solution. Besides, the suction effect thickens the boundary layer, increasing the shear stress and $f^{\prime \prime}(0)$, as seen in Figure 2. Furthermore, Figure 13 depicts the effect of parameter $s$ on $\theta(\eta)$ for the shrinking case. The result shows that when $s$ rises, the temperature distribution decreases, reducing the thickness of the thermal boundary layer. 
Moreover, these profiles are shown in Figure 12 and 13 that meet the boundary requirements asymptotically, supporting the obtained outcomes and the occurrence of the dual solutions.

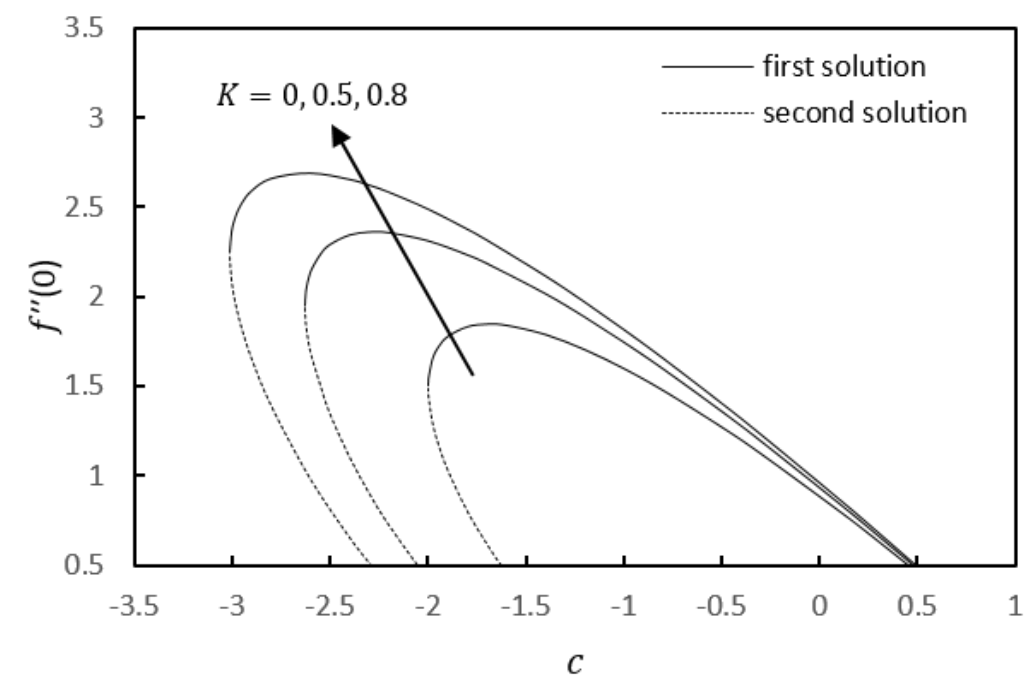

Fig. 10. Skin friction coefficient $f^{\prime \prime}(0)$ with $c$ when $\operatorname{Pr}=1, s=$ 0.3 and $A=B=0.5$

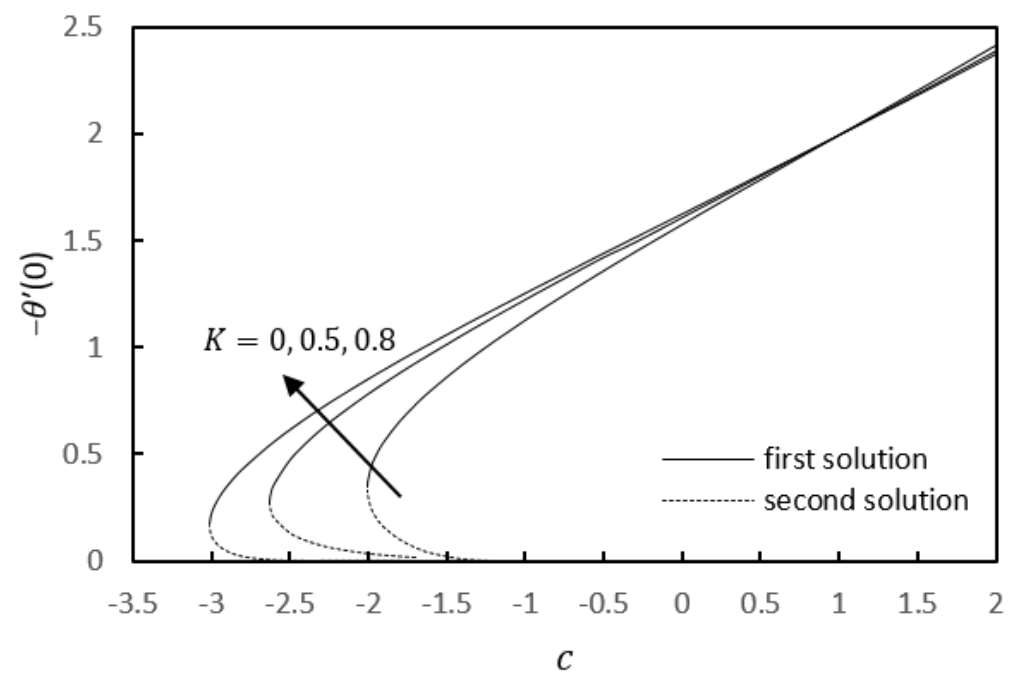

Fig. 11. Local Nusselt number $-\theta^{\prime}(0)$ with $c$ when $\operatorname{Pr}=1$, $s=0.3$ and $A=B=0.5$ 


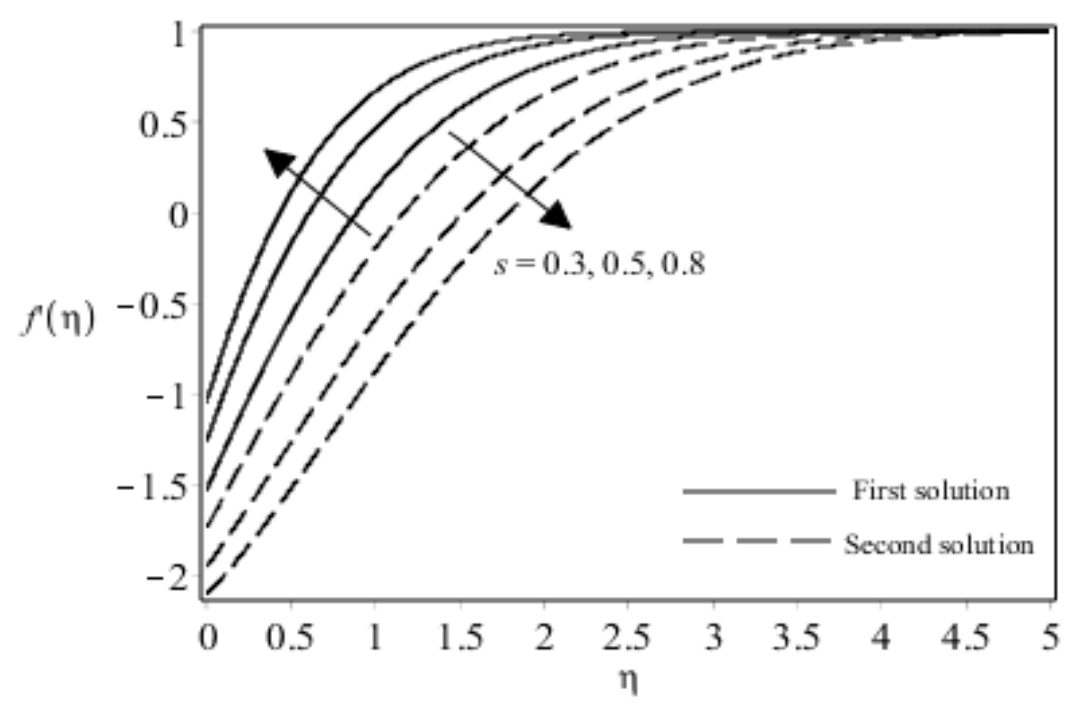

Fig. 12. Velocity profiles $f^{\prime}(\eta)$ for different values of $s>0$ when $\operatorname{Pr}=1, K=A=B=0.5$ and $c=-2.6$ (shrinking)

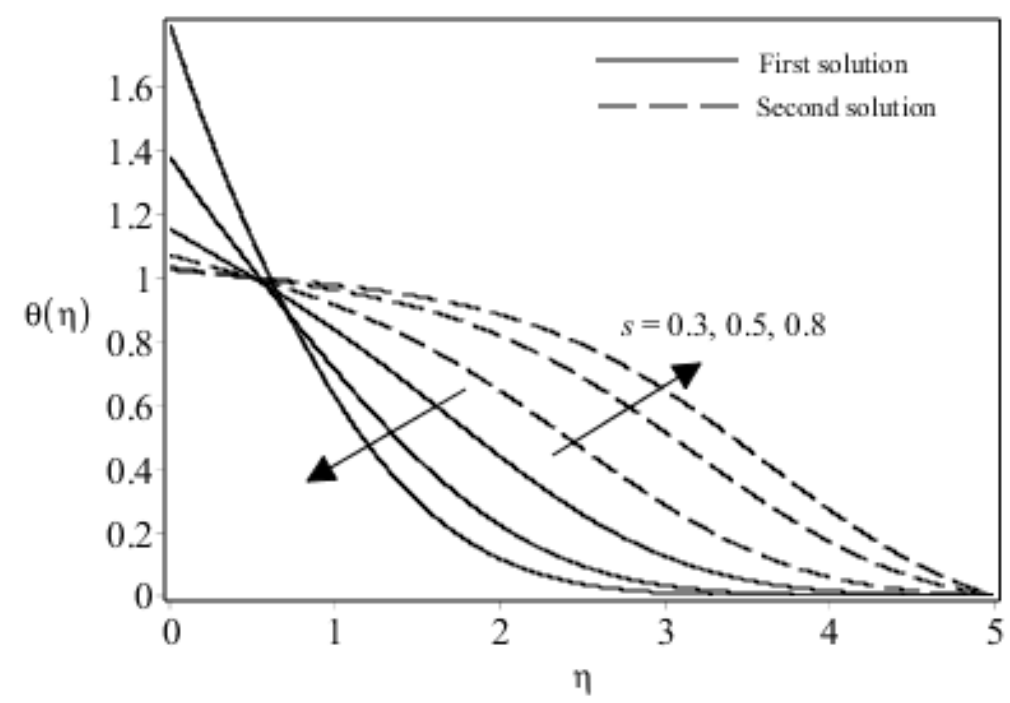

Fig. 13. Temperature profiles $\theta(\eta)$ for different values of $s>0$ when $\operatorname{Pr}=1, K=A=B=0.5$ and $c=-2.6$ (shrinking)

Figure 14 and 15 illustrate the $f^{\prime}(\eta)$ and $\theta(\eta)$ profiles for several injection parameter $s$ values in the shrinking case. As shown in Figure 14, as the injection parameter decreases, the thickness of the boundary layer decrease in the first solution while the opposite manner in the second solution. In Figure 15, it is shown that a decrease in injection parameters will increase the thermal boundary thickness as illustrated in the first solution. Thus, the injection effect reduces the temperature gradient and hence decrease the heat transfer rate on the surface. Based on Figure 14 to 15, it should be emphasised that these profiles fulfil the far-field boundary conditions, confirming the accuracy of the findings and the duality of the solutions. Figure 16 and 17 display the $f^{\prime}(\eta)$ and $\theta(\eta)$ profiles for some values of parameter $A$ in the shrinking case. The first solution, seen in Figure 16, exhibits a rise in velocity as the parameter $A$ rises, whereas the second solution exhibits the opposite effect. In Figure 17, as the parameter $A$ values increase, the first solution decreases while the second solution increases. Moreover, the thickness of the velocity boundary layer is higher in the first solution than in the second solution, but the thermal boundary layer thickness is reversed. 


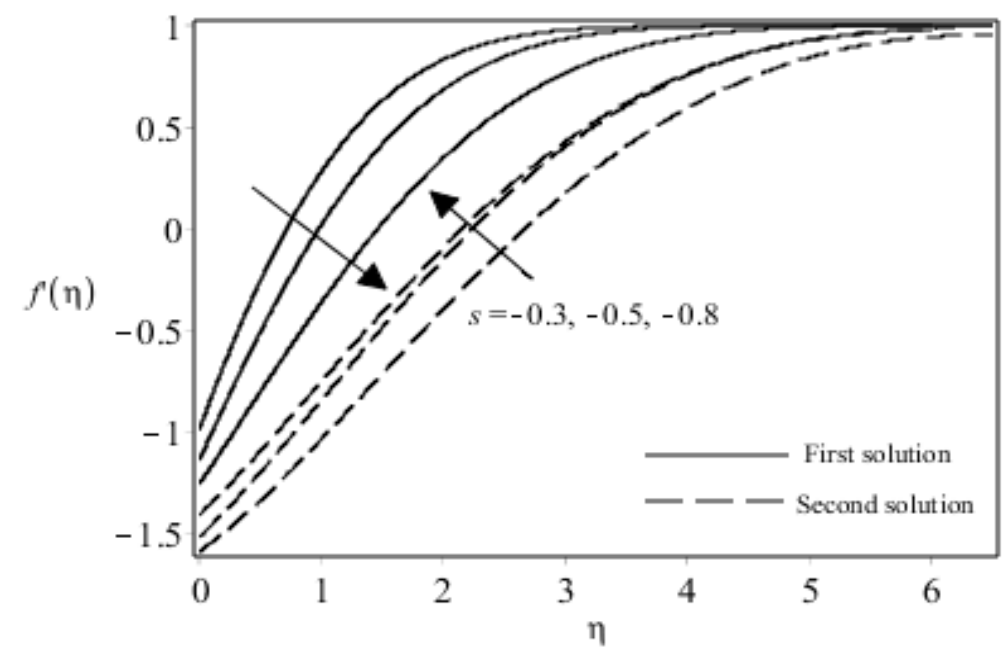

Fig. 14. Velocity profiles $f^{\prime}(\eta)$ for different values of $s<0$ when $\operatorname{Pr}=1, K=A=B=0.5$ and $c=-1.7$ (shrinking)

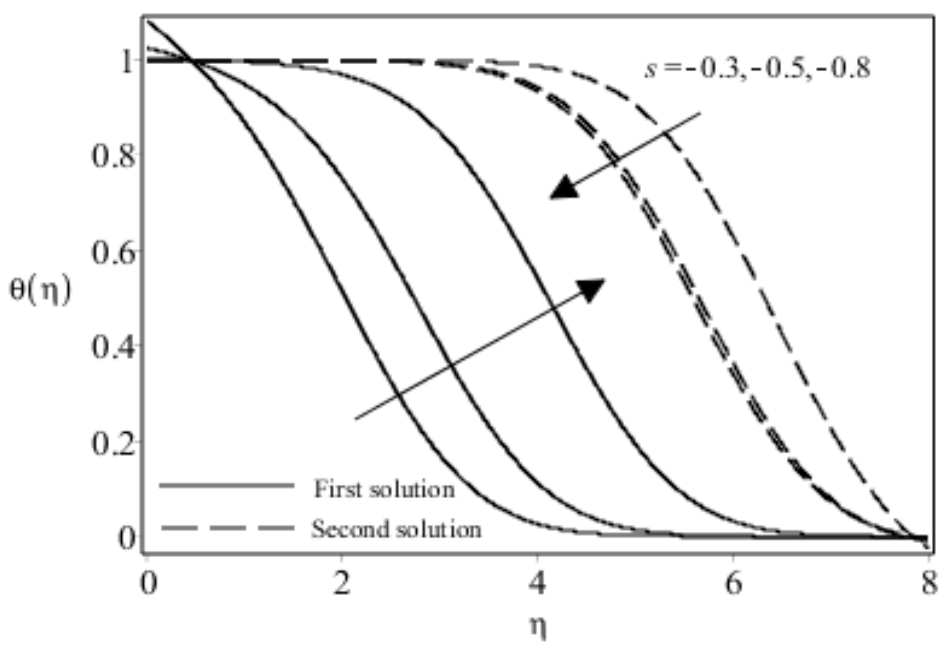

Fig. 15. Temperature profiles $\theta(\eta)$ for different values of $s<$ 0 when $\operatorname{Pr}=1, K=A=B=0.5$ and $c=-1.7$ (shrinking)

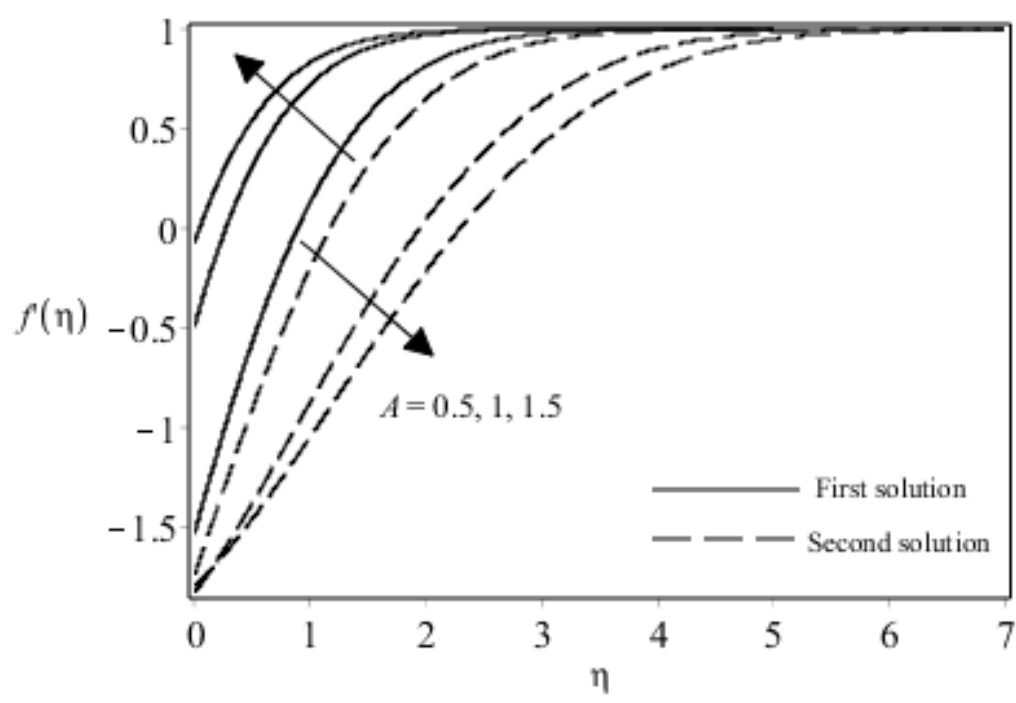

Fig. 16. Velocity profiles $f^{\prime}(\eta)$ for different values of $A$ when $\operatorname{Pr}=1, s=0.3, K=B=0.5$ and $c=-2.6$ (shrinking) 


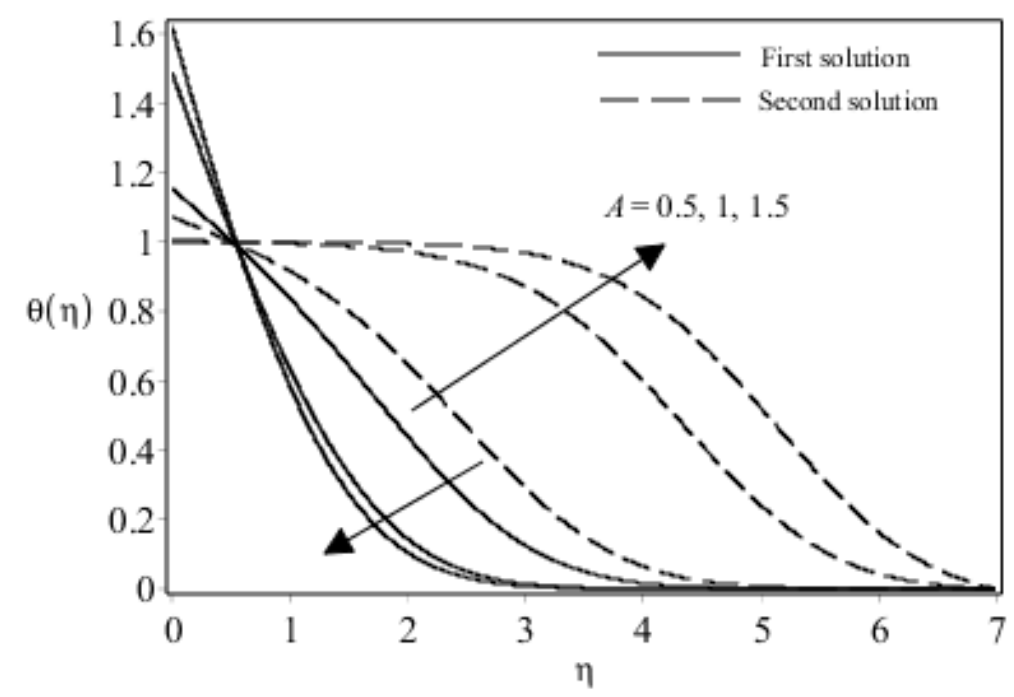

Fig. 17. Temperature profiles $\theta(\eta)$ for different values of $A$ when $\operatorname{Pr}=1, s=0.3, K=B=0.5$ and $c=-2.6$ (shrinking)

Whereas, Figure 18 and 19 demonstrated the variation of the velocity and temperature profiles for various thermal slip parameter values in the shrinking case. However, it can be observed that the velocity values remain constant as thermal slip increases. This corroborates the findings in Figure 8 , which demonstrate that thermal slip does not influence the fluctuation of the skin friction coefficient. In Figure 19, the temperature profiles increase with the thermal slip parameters up until a certain point before it approaches zero. Finally, Figure 20 and 21 show the velocity and temperature profiles for various values of the permeability parameter $K$, with other parameters being constant. The velocity drops as the permeability parameter $K$ rises, while the temperature distributions do the reverse. Temperature is more sensitive to the permeability parameter than the velocity profiles, as seen in the figures. From a physical viewpoint, it demonstrates that when suction, velocity slip, and porosity parameters rise, the fluid's velocity increases. Moreover, all of these profiles were satisfied asymptotically under the boundary conditions. Therefore, the precision of this research's numerical findings will be increased.

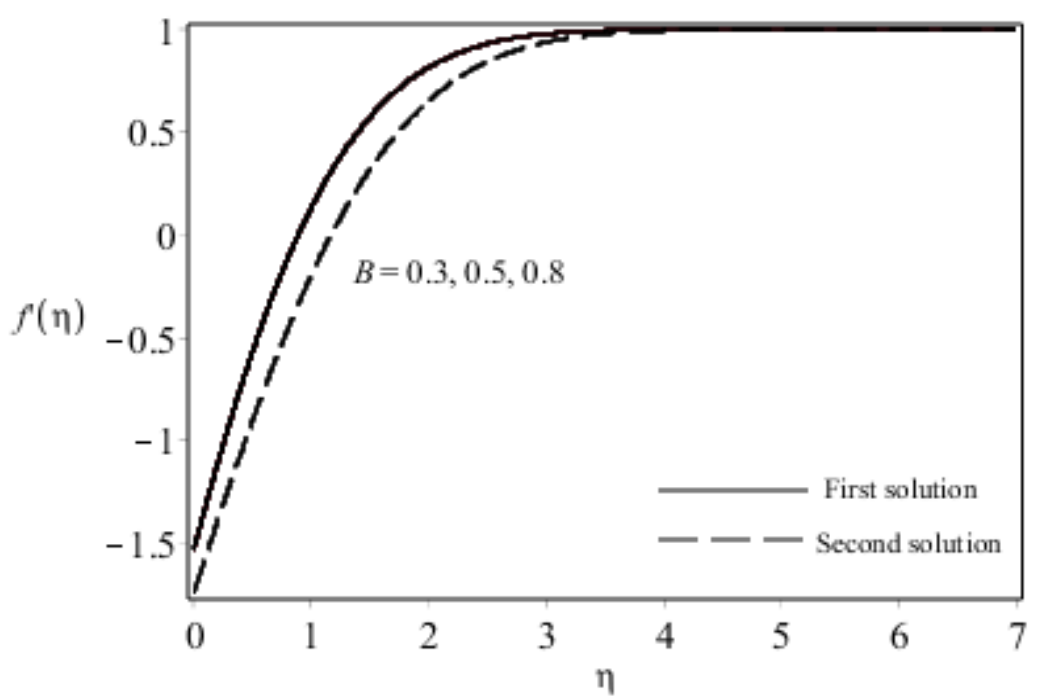

Fig. 18. Velocity profiles $f^{\prime}(\eta)$ for different values of $B$ when $\operatorname{Pr}=1, s=0.3, K=B=0.5$ and $c=-2.6$ (shrinking) 


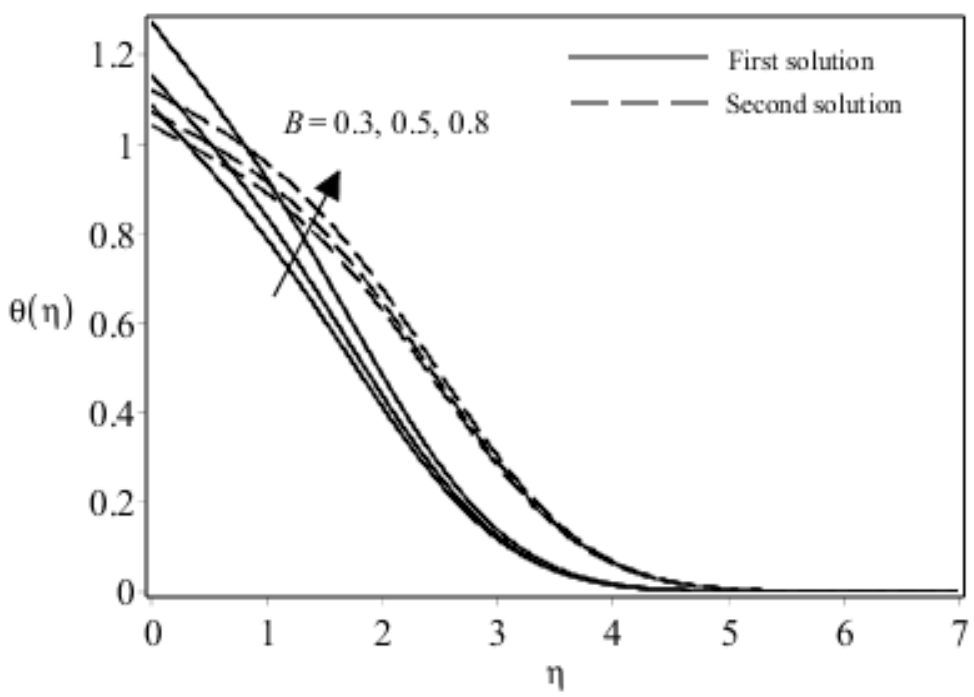

Fig. 19. Temperature profiles $\theta(\eta)$ for different values of $A$ when $\operatorname{Pr}=1, s=0.3, K=B=0.5$ and $c=-2.6$ (shrinking)

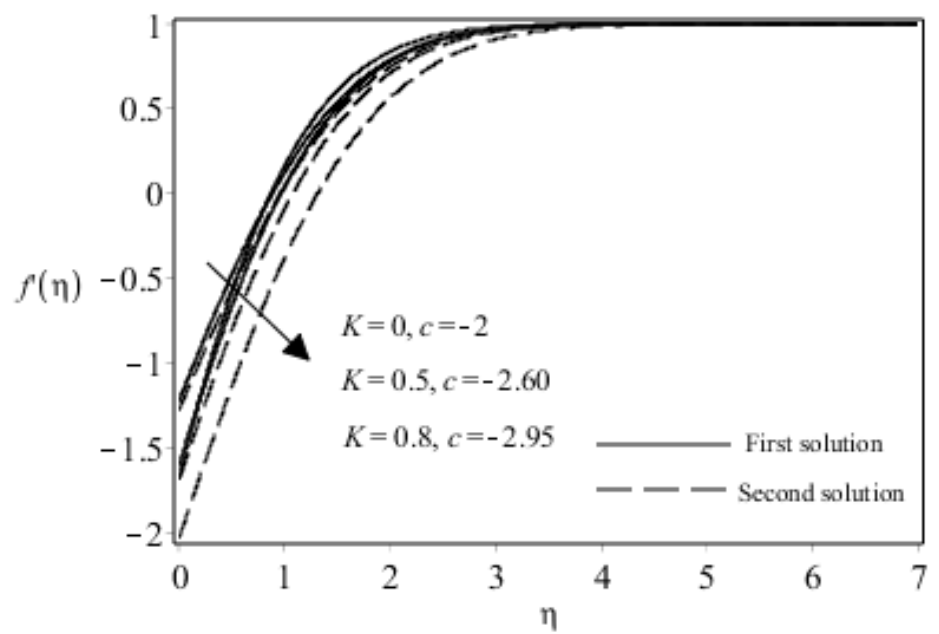

Fig. 20. Velocity profiles $f^{\prime}(\eta)$ for different values of $K$ and $c$ when $\operatorname{Pr}=1, A=B=0.5$ and $s=0.3$

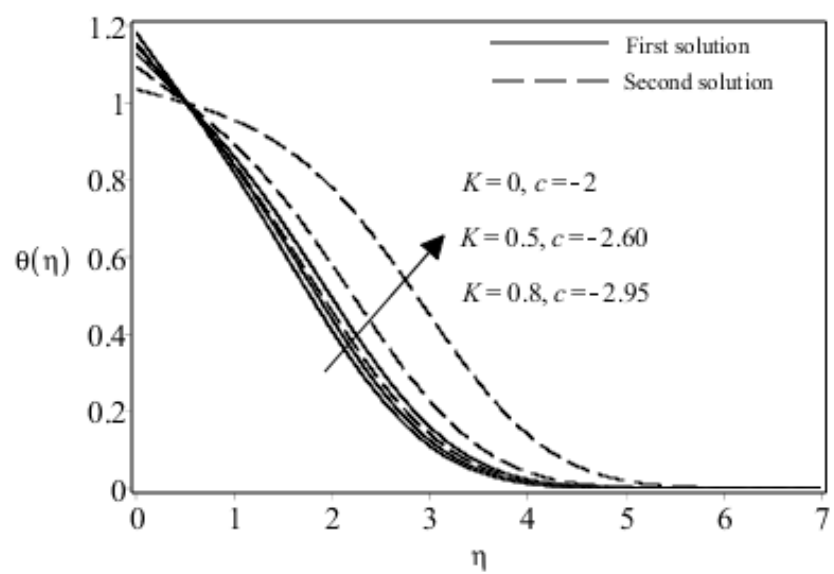

Fig. 21. Temperature profiles $\theta(\eta)$ for different values of $K$ and $c$ when $\operatorname{Pr}=1, A=B=0.5$ and $s=0.3$ 


\section{Conclusions}

A numerical analysis of stagnation point flow and heat transfer over a shrinking sheet with the impact of slip and suction through a porous medium is presented in this article. The equations controlling the boundary layer were numerically solved using the shooting technique. The skin friction coefficient and the local Nusselt number were determined and analysed in relation to the suction, injection, slip, and permeability parameters, as well as the velocity and temperature profiles. This research discovered the following

i. Unique solution occurs in the stretching case $(c>0)$ and dual solutions occur in the shrinking case $\left(c_{c}<c<0\right)$

ii. As suction, velocity slip, and permeability parameter values rise, the skin friction coefficient and local Nusselt number increase as well

iii. The skin friction coefficient and the boundary layer separation does not influence by the thermal slip parameter

iv. As the injection parameter increases, the skin friction coefficient and local Nusselt number decrease

v. The solutions domain become larger in the presence of the suction effect, velocity slip and permeability parameter

\section{Acknowledgement}

The authors would want to express their appreciation to the reviewers and editors for their insightful remarks and recommendations, which helped to enhance this work. The Ministry of Higher Education (MOHE) funded this study via the Fundamental Research Grant Scheme (FRGS/1/2016/STG06/UPM/03/1).

\section{References}

[1] Chamkha, Ali J., and Abdul-Rahim A. Khaled. "Similarity solutions for hydromagnetic mixed convection heat and mass transfer for Hiemenz flow through porous media." International Journal of Numerical Methods for Heat \& Fluid Flow (2000). https://doi.org/10.1108/09615530010306939

[2] Crane, Lawrence J. "Flow past a stretching plate." Zeitschrift für angewandte Mathematik und Physik ZAMP 21 , no. 4 (1970): 645-647. https://doi.org/10.1007/BF01587695

[3] Gupta, P. S., and A. S. Gupta. "Heat and mass transfer on a stretching sheet with suction or blowing." The Canadian journal of chemical engineering 55, no. 6 (1977): 744-746. https://doi.org/10.1002/cjce.5450550619

[4] Wang, C. Y. "Stagnation flow towards a shrinking sheet." International Journal of Non-Linear Mechanics 43, no. 5 (2008): 377-382. https://doi.org/10.1016/i.ijnonlinmec.2007.12.021

[5] Bhattacharyya, S. N., and A. S. Gupta. "On the stability of viscous flow over a stretching sheet." Quarterly of applied mathematics 43, no. 3 (1985): 359-367. https://doi.org/10.1090/qam/814233

[6] TC, Chiam. "Stagnation-point flow towards a stretching plate." Journal of the physical society of Japan 63, no. 6 (1994): 2443-2444. https://doi.org/10.1143/JPSJ.63.2443

[7] Mahapatra, T. Ray, and A. S. Gupta. "Heat transfer in stagnation-point flow towards a stretching sheet." Heat and Mass transfer 38, no. 6 (2002): 517-521. https://doi.org/10.1007/s002310100215

[8] Ishak, Anuar, Roslinda Nazar, and loan Pop. "Mixed convection boundary layers in the stagnation-point flow toward a stretching vertical sheet." Meccanica 41, no. 5 (2006): 509-518. https://doi.org/10.1007/s11012-006-0009-4

[9] Layek, G. C., S. Mukhopadhyay, and Sk A. Samad. "Heat and mass transfer analysis for boundary layer stagnationpoint flow towards a heated porous stretching sheet with heat absorption/generation and suction/blowing." International communications in heat and mass transfer 34, no. 3 (2007): $347-356$. https://doi.org/10.1016/j.icheatmasstransfer.2006.11.011

[10] Nadeem, S., Anwar Hussain, and Majid Khan. "HAM solutions for boundary layer flow in the region of the stagnation point towards a stretching sheet." Communications in Nonlinear Science and Numerical Simulation 15, no. 3 (2010): 475-481. https://doi.org/10.1016/i.cnsns.2009.04.037 
[11] Mustafa, M., T. Hayat, I. Pop, S. Asghar, and S. Obaidat. "Stagnation-point flow of a nanofluid towards a stretching sheet." International Journal of Heat and Mass Transfer 54, no. 25-26 (2011): 5588-5594. https://doi.org/10.1016/j.ijheatmasstransfer.2011.07.021

[12] Ishak, Anuar, Yian Yian Lok, and loan Pop. "Stagnation-point flow over a shrinking sheet in a micropolar fluid." Chemical Engineering Communications 197, no. $11 \quad$ (2010): 1417-1427. https://doi.org/10.1080/00986441003626169

[13] Bachok, Norfifah, Anuar Ishak, and loan Pop. "Boundary layer stagnation-point flow toward a stretching/shrinking sheet in a nanofluid." Journal of heat transfer 135, no. 5 (2013). https://doi.org/10.1115/1.4023303

[14] Bhattacharyya, Krishnendu, Swati Mukhopadhyay, and G. C. Layek. "Slip effects on boundary layer stagnation-point flow and heat transfer towards a shrinking sheet." International Journal of Heat and Mass Transfer 54, no. 1-3 (2011): 308-313. https://doi.org/10.1016/j.ijheatmasstransfer.2010.09.041

[15] Bhattacharyya, Krishnendu, and G. C. Layek. "Effects of suction/blowing on steady boundary layer stagnation-point flow and heat transfer towards a shrinking sheet with thermal radiation." International Journal of Heat and Mass Transfer 54, no. 1-3 (2011): 302-307. https://doi.org/10.1016/i.ijheatmasstransfer.2010.09.043

[16] Bhattacharyya, Krishnendu. "Dual solutions in boundary layer stagnation-point flow and mass transfer with chemical reaction past a stretching/shrinking sheet." International Communications in Heat and Mass Transfer 38, no. 7 (2011): 917-922. https://doi.org/10.1016/i.icheatmasstransfer.2011.04.020

[17] Bhattacharyya, Krishnendu. "Dual solutions in unsteady stagnation-point flow over a shrinking sheet." Chinese Physics Letters 28, no. 8 (2011): 084702. https://doi.org/10.1088/0256-307X/28/8/084702

[18] Bhattacharyya, Krishnendu. "Heat transfer analysis in unsteady boundary layer stagnation-point flow towards a shrinking/stretching sheet." Ain Shams Engineering Journal 4, no. $2 \quad$ (2013): $259-264$. https://doi.org/10.1016/i.asej.2012.07.002

[19] Bhattacharyya, Krishnendu, Swati Mukhopadhyay, G. C. Layek, and Ioan Pop. "Effects of thermal radiation on micropolar fluid flow and heat transfer over a porous shrinking sheet." International Journal of Heat and Mass Transfer 55, no. 11-12 (2012): 2945-2952. https://doi.org/10.1016/i.ijheatmasstransfer.2012.01.051

[20] Bhattacharyya, Krishnendu, and Kuppalapalle Vajravelu. "Stagnation-point flow and heat transfer over an exponentially shrinking sheet." Communications in Nonlinear Science and Numerical Simulation 17, no. 7 (2012): 2728-2734. https://doi.org/10.1016/i.cnsns.2011.11.011

[21] Bhattacharyya, Krishendu. "Boundary layer stagnation-point flow of casson fluid and heat transfer towards a shrinking/stretching sheet." Frontiers in Heat and Mass Transfer (FHMT) 4, no. 2 (2013). https://doi.org/10.5098/hmt.v4.2.3003

[22] Bhattacharyya, Krishnendu, Kuppalapalle Vajravelu, and Tasawar Hayat. "Slip effects on the parametric space and the solution for boundary layer flow of casson fluid over a porous stretching/shrinking sheet." International Journal of Fluid Mechanics Research 40, no. 6 (2013). https://doi.org/10.1615/InterJFluidMechRes.v40.i6.20

[23] Bhattacharyya, Krishnendu. "Heat transfer in boundary layer stagnation-point flow towards a shrinking sheet with non-uniform heat flux." Chinese Physics B 22, no. 7 (2013): 074705. https://doi.org/10.1088/1674$\underline{1056 / 22 / 7 / 074705}$

[24] Bhattacharyya, Krishnendu, G. C. Layek, and G. S. Seth. "Soret and Dufour effects on convective heat and mass transfer in stagnation-point flow towards a shrinking surface." Physica Scripta 89, no. 9 (2014): 095203. https://doi.org/10.1088/0031-8949/89/9/095203

[25] Aman, Fazlina, Anuar Ishak, and Ioan Pop. "Magnetohydrodynamic stagnation-point flow towards a stretching/shrinking sheet with slip effects." International Communications in Heat and Mass Transfer 47 (2013): 68-72. https://doi.org/10.1016/i.icheatmasstransfer.2013.06.005

[26] Rosali, Haliza, Anuar Ishak, and Ioan Pop. "Stagnation point flow and heat transfer over a stretching/shrinking sheet in a porous medium." International Communications in Heat and Mass Transfer 38, no. 8 (2011): 1029-1032. https://doi.org/10.1016/j.icheatmasstransfer.2011.04.031

[27] Pal, Dulal, and Gopinath Mandal. "Mixed convection-radiation on stagnation-point flow of nanofluids over a stretching/shrinking sheet in a porous medium with heat generation and viscous dissipation." Journal of Petroleum Science and Engineering 126 (2015): 16-25. https://doi.org/10.1016/i.petrol.2014.12.006

[28] Bakar, Shahirah Abu, Norihan Md Arifin, R. Nazar, Fadzilah Md Ali, and Ioan Pop. "Forced convection boundary layer stagnation-point flow in Darcy-Forchheimer porous medium past a shrinking sheet." Frontiers in Heat and Mass Transfer 7 (2016): 38. https://doi.org/10.5098/hmt.7.38

[29] Bhattacharyya, K., and S. Shafie. "Effect of partial slip on an unsteady MHD mixed convection stagnation-point flow of a micropolar fluid towards a permeable shrinking sheet." Alexandria Engineering Journal 55, no. 2 (2016): 12851293. https://doi.org/10.1016/i.aej.2016.04.018 
[30] Nasir, Nor Ain Azeany Mohd, Anuar Ishak, and loan Pop. "Stagnation-point flow and heat transfer past a permeable quadratically stretching/shrinking sheet." Chinese Journal of Physics 55, no. 5 (2017): 2081-2091. https://doi.org/10.1016/j.cjph.2017.08.023

[31] Kumar, Rakesh, and Shilpa Sood. "Numerical analysis of stagnation point nonlinear convection flow through porous medium over a shrinking sheet." International Journal of Applied and Computational Mathematics 3, no. 2 (2017): 971-985. https://doi.org/10.1007/s40819-016-0150-2

[32] Shomali, Ali, Abbas Panahi, Mohammad Hossein Sabour, and Meysam Mosania. "Unsteady stagnation point flow of nanofluid over a stretching/shrinking sheet in a porous medium." In 5th International Conference of Fluid Flow, Heat and Mass Transfer Proceedings, pp. 1-10. 2018. https://doi.org/10.11159/ffhmt18.117

[33] Jamaludin, Anuar, and Roslinda Nazar. "Dual solutions of stagnation-point flow over an exponentially stretching/shrinking sheet in a porous medium with suction and velocity slip: A stability analysis." In Journal of Physics: Conference Series, vol. 1212, no. 1, p. 012026. IOP Publishing, 2019. https://doi.org/10.1088/1742$6596 / 1212 / 1 / 012026$

[34] Japili, Nirwana, Haliza Rosali, and Norfifah Bachok. "Suction Effect on Stagnation Point Flow and Heat Transfer over an Exponentially Shrinking Sheet in a Porous Medium." Journal of Advanced Research in Fluid Mechanics and Thermal Sciences 73, no. 2 (2020): 163-174. https://doi.org/10.37934/arfmts.73.2.163174

[35] Rosali, Haliza, Mohd Noor Badlilshah, Mohamat Aidil Mohamat Johari, and Norfifah Bachok. "Unsteady Boundary Layer Stagnation Point Flow and Heat Transfer over a Stretching Sheet in a Porous Medium with Slip Effects." CFD Letters 12, no. 10 (2020): 52-61. https://doi.org/10.37934/cfdl.12.10.5261

[36] Norzawary, Nur Hazirah Adilla, Norfifah Bachok, and Fadzilah Md Ali. "Stagnation Point Flow over a Stretching/shrinking Sheet in a Carbon Nanotubes with Suction/Injection Effects." CFD Letters 12, no. 2 (2020): 106114.

[37] Rosali, Haliza, and Anuar Ishak. "Stagnation-point flow over a stretching/shrinking sheet in a porous medium." In AIP Conference Proceedings, vol. 1571, no. 1, pp. 949-955. American Institute of Physics, 2013. https://doi.org/10.1063/1.4858776 